psychiatrists from the University of Milan from surrounding northern Italian communities (3 families). All individuals were evaluated by a trained clinician. Subjects were interviewed using a modified SADS structured format (Schedule for Affective Disorders and Schizophrenia; Endicott \& Spitzer 1978). Records from previous hospitalisations and of psychiatric treatments were obtained, and further information was collected on each individual from reliable family members. Diagnoses were made, based on information from these multiple sources, using DSM-III-R (American Psychiatric Association, 1987) criteria. None of these patients had any atypical movements. Ethical approval has been granted for the use of these samples for linkage analysis and for the investigation of candidate genes. The CAG repeats in the B37 genes from 55 affected probands were measured. Polymerase chain reactions were performed essentially as described by Koide et al (1994) except that $25 \mathrm{ng}$ of each primer was used and $64^{\circ} \mathrm{C}$ was used as the annealing temperature.

These patients had between 8 and 19 repeats, clearly within the reported normal ranges (Koide et al, 1994: Nagafuchi et al, 1994), and had an observed heterozygosity of $73 \%$ that was significantly different to that observed in Japanese ( $88 \%$ $\left(\chi^{2}=6.502, P<0.025\right)$; Koide et al, 1994). It is possible that some of the patients who appeared to be homozygotes for these B37 repeats were in fact heterozygotes with very large mutant alleles that were not successfully amplified by our PCR however, we have been able to amplify 60 repeats at this locus and see the results after an overnight exposure of the autoradiograph. In addition, a nuclear family with two schizophrenic brothers was analysed separately for B37 expansions. Both brothers had severe abnormal choreiform movements of unknown aetiology and were thus candidates for DRPLA. However, they too had normal numbers of $B 37$ repeats. Thus, we conclude that abnormal expansions in the B37 gene are not found in most schizophrenic patients.

We thank Drs N. Bass, G. Shields, A. Boccio-Smith, C. Morganti and $A$. Vita for helping with family evaluations. Funding for the clinical collections of families and preparation of DNA was provided by a NIMHR01-844245 in the USA and the Medical Research Council in the UK. We are also grateful to the Huntington's Disease Association (UK) for research funding.

ENDICOTT, J. \& SPITzER, R. L. (1978) A diagnostic interview: the SADS. Archives of General Psychiatry, 35, 837-853.

Kendler, K. S. \& Difhl, S. R. (1993) The genetics of schizophrenia: a current, genetic-epidemiologic perspective. Schizophrenia Bulletin. 19, 261-285.
KoIDE, R., IKEUCHI, T., ONODERA, O., et al (1994) Unstable expansion of CAG repeat in hereditary dentatorubralpallidoluysian atrophy (DRPLA). Nature Genetics, 6, 9-12.

Nagafuchi, S., Yanagisawa, H., Sato, K., et al (1994) Dentatorubral and pallidolusian atrophy expansion of an unstable CAG trinucleotide on chromosome 12p. Nature Genetics, 6, $14-18$.

Ross, C. A., McInNis, M. G., Margolis, R. L., et al (1993) Genes with triplet repeats: candidate mediators of neuropsychiatric disorders. Trends in Neurosciences, 16, 254-260.

D. C. RubinszTEIN

J. LEGGO

S. GOODBURN

D. E. BARTON

M. A. Ferguson-Smith

East Anglian Regional Genetics Service

Box 158, Addenbrooke's NHS Trust

Cambridge CB2 $2 Q Q$

C. A. Ross

S. H. LI

Ross 618, Johns Hopkins University

720 Rutland Avenue

Baltimore

Maryland 21205-2196, USA

R. LOFTHOUSE

T. J. Crow

Clinical Research Center

Watford Road

Harrow

Health Sciences Center

L. E. DELISI

State University of New York at Stonybrook

New York 11794, USA

Fluvoxamine - prescription event monitoring

SIR: Edwards et al (BJP, March 1994, 164, 387 395) provided valuable additional safety data for fluvoxamine in their prescription event monitoring (PEM) study.

Fluvoxamine has been available in the UK since 1987 and an estimated 6 million patients have been treated worldwide. Data from 10401 patients in the PEM study gave a side-effect profile comparable with the Duphar clinical trials safety database of 24624 patients (Wagner et al, 1992), recently updated to a total of 34587 patients (unpublished). Gastrointestinal side-effects predominated in both data sets with only nausea/vomiting reported at an incidence greater than $10 \%$, diminishing rapidly after the first month. No new adverse effects were identified and the incidence of seizures was shown to be no higher on than off treatment. This should provide considerable reassurance to prescribers. 
These data put into perspective the often quoted established 'safety' of the tricyclic antidepressants (TCAs), many of which were first available before or in the infancy of regulatory drug monitoring schemes or post-marketing surveillance studies. There are no similar bodies of safety data for most of the TCAs and older antidepressants; their 'established' safety results more from familiarity than hard data.

However, while PEM is an important addition to drug safety monitoring methodology, it cannot assess efficacy. General practitioners were asked to record on the follow-up form sent one year after the initial prescription, a simple question: was the drug effective? Apparently most GPs felt that fluvoxamine was not effective, yet only $38.4 \%$ of the sub-group analysed for the reason for stopping treatment recorded lack of efficacy as a factor. This is clearly a discrepant result. A number of reasons for this supposed low efficacy were discussed - all may be relevant, but the most significant is that PEM was not designed to study efficacy.

Efficacy is established in carefully designed and controlled double-blind studies using trained raters and specialised rating scales. Fluvoxamine has consistently been shown to be superior to placebo and to be as effective as other antidepressants (Wilde et al, 1993). Efficacy claims based on a PEM study would have been rightly criticised. Inferences regarding lack of efficacy are no more credible.

WAGNer, W., PlekkenPol, B., Gray, T. E., et al (1992) Review of fluvoxamine safety database. Drugs, 43 (suppl. 2), 48-54.

WILde, M. I., Plosker, G. L. \& BenfIELD, P. (1993) Fluvoxamine: an updated review of its pharmacology, and therapeutic use in depressive illness. Drugs, 46, 895-924.

\section{J. J. ASHFORD}

Duphar Laboratories Ltd

Southampton SO18 3JD

\section{Death during alcohol withdrawal}

SIR: Circulatory collapse in delirium tremens or death during an alcohol withdrawal seizure are rare, but still occur. Much can be done to prevent this occurring by timely prescribing. Nurses giving sedation for alcohol withdrawal should be alert to the possibility that a dose may be vomited, in which case parenteral benzodiazepines or rectal diazepam may be indicated, particularly in a patient with a history of withdrawal seizures.

The typical ward regime of 6-hourly dosage may mean that a patient who had his last drink 12 hours before admission may go almost 18 hours before receiving his first dose of sedation. The nurse should be instructed to give the first dose at admission in most patients. An 'as required' dose enables the nurse to titrate dosage against symptoms.

Medication can be given while there is still some alcohol in the body, but not to a patient who is still intoxicated and unable to give true consent, and make a contract about the treatment plan. This applies if detoxification is to be carried out as an in-patient or as an out-patient. In out-patients, medication could be issued to the community nurse or relative, to commence when the patient is mentally clear enough to agree not to take alcohol.

There are serious risks in under-medicating individuals severely dependent on alcohol who precipitously cease alcohol intake for whatever reason.

Royal Edinburgh Hospital

J. D. CHICK

35 Morningside Park

Edinburgh EHIO SHD

\section{Glucocorticoids and the genesis of depressive illness}

SIR: Dinan's integration of possible secondary neuroendocrine findings in depression supports the central role of glucocorticoids in its pathogenesis (BJP, March 1994, 164, 365-371). The neuroendocrine changes quoted, and others not mentioned, specifically blunted hypoglycaemic response to the insulin stress test and subtle changes in the hypothalamo-pituitary-gonadal axis, are one of many areas of depressive phenomenology which may be secondary to hypercortisolaemia. High levels of exogenous steroids cause high intracellular sodium concentrations and eosinophilia, both of which have long been recognised in depression. Some evidence suggests that the characteristic sleep disturbances which are seen in both depression and Cushing's syndrome may be mediated by glucocorticoids (Shipley et al, 1992). A number of independent authors have documented that hypothalamo-pituitary adrenal (HPA) hyperactivity is associated with specific clinical features including psychomotor retardation, weight loss and cognitive decline (Miller \& Nelson, 1987). High levels of cortisol have also been closely related to a variety of neuroanatomical changes including hippocampal atrophy which may be an important mechanism of steroid-related psychopathological change (Hauser, 1991).

While these associations only provide circumstantial support for the involvement of glucocorticoids in the pathogenesis of depressive disorders, recent research is more convincing. HPA axis status 